\title{
RECURRENT SPINAL HYDATIDOSIS - A CASE REPORT WITH 3 YEARS FOLLOW UP AND REVIEW OF LITERATURE
}

\author{
Avinash Parthasarathy ${ }^{1}$, Chidanand K. J. C2 , Prakashappa T. $\mathrm{H}^{3}$
}

\section{HOW TO CITE THIS ARTICLE:}

Avinash Parthasarathy, Chidanand K. J. C, Prakashappa T. H. "Recurrent Spinal Hydatidosis - A Case Report with 3 Years Follow up and Review of Literature". Journal of Evolution of Medical and Dental Sciences 2014; Vol. 3, Issue 20, May 19; Page: 5546-5551, DOI: 10.14260/jemds/2014/2627

ABSTRACT: Spinal hydatid cyst, constituting 1\% - $2.5 \%$ of the total cases of hydatid disease, is a rare form of hydatid disease. We present a case of pathologically confirmed primary intradural and extradural spinal hydatid cyst in an otherwise healthy patient causing spinal cord compression. The patient had atypical symptoms on presentation with sudden onset of paraplegia and hence, the diagnosis was not established pre-operatively. The patient underwent spinal decompression surgery resulting in complete recovery. However there was a recurrence at the 24 months follow-up for which she was treated in a similar fashion.

KEYWORDS: Spine, hydatid disease, spinal cord compression.

INTRODUCTION: Hydatid cyst infestation, or echinococcosis, is one of the oldest diseases known to man. Human beings are accidental hosts in the life cycle of Echinococcus granulosum, the worm responsible for hydatid disease. ${ }^{1}$ Bone hydatidosis is rare in relation to the infestation of other organs and occurs in $1 \%$ to $2.5 \%$ of cases of human hydatidosis, out of which $50 \%$ are seen in the spine. ${ }^{2}$

In hydatid disease of the vertebrae, the parasites spread along the bony intratrabecular space, destroying the bone. However, intervertebral discs are usually preserved because the disease tends to propagate beneath the periosteum and ligaments. ${ }^{1}$ It is a common cause of spinal cord compression in endemic areas and the diagnosis remains obscure until symptoms resulting from complications due to root and cord compression appear. Surgical decompression and stabilization, combined with adjuvant chemotherapy, is the treatment of choice. ${ }^{3}$ We report a case of recurrent spinal hydatidosis and its management with a follow-up of three years.

CASE REPORT: A 20 year old lady presented to us with a sudden onset of weakness in both her lower limbs since 10 days. There were no preceding symptoms, and a physical and neurological examination revealed total paraplegia [spastic]. A thoraco-lumbar (TL) spine radiograph at initial evaluation showed no abnormality.

Routine blood investigations were within normal limits. Magnetic resonance imaging (MRI) demonstrated an extradural and extra spinal fluid filled mass with involvement of the body, pedicle and posterior arch of D7 vertebra, with a possible diagnosis of neoplasm, infection or tuberculosis (Figure 1A and 1B). The MRI did not demonstrate any other visceral involvement.

When the patient was taken up for surgical decompression of the spine, we found a cyst filled with clear fluid which moved with respiration. During excision of the cyst, it burst open and expelled clear and colorless fluid. The cyst was removed and washed with hypertonic saline. Due precautions were taken to prevent an anaphylactic reaction. Histo-pathological examination confirmed a diagnosis of a hydatid cyst. The patient showed progressive recovery and was capable of an independent life with no muscular or neural deficits. She was discharged with the advice of tablet 
albendazole [400 mg. twice daily]. One month later she came with a discharging sinus over the 9th rib, which was diagnosed to be an infected cyst and was excised (Figure 2). Three months later she again presented with a mild fever and back ache.

A thoraco-lumbar (TL) spine MRI revealed paravertebral cystic cavities with involvement of three vertebrae. However, there was no intra-spinal collection (Figure 3A and 3B). A Computed Tomography (CT) guided aspiration yielded pus and the culture and sensitivity report confirmed enterobacter and pseudomonas sensitive to ciprofloxacin and amikacin. In addition to a course of ciprofloxacin and amikacin to address the secondary infection, she was advised to continue taking albendazole.

She was apparently well for a further two years after which she started experiencing gradual onset progressive weakness in both the lower limbs. The MRI showed cysts in the spinal canal, which were compressing the cord at D 5 and D 6 level (Figure 4A and 4B). The patient again underwent surgery, the cyst found and excised and the surrounding tissues washed with hypertonic saline. She was instructed to wear a hyperextension brace, as there was wedge compression of the D 6 vertebra. At the last follow-up of three years, she was able to carry out her routine activities independently.

DISCUSSION: The spine is the most common location of bone hydatidosis. ${ }^{4}$ The diagnosis is often difficult as the symptoms of spinal hydatid disease are not typical and the usual presentation is of neural compromise. ${ }^{1,5,6}$ Generally, the first symptoms are back pain and radicular pain. Weakness of the limbs occurs in the later phase of the disease, and paraplegia has been reported in $25 \%$ to $84 \%$ of cases. $^{7}$

Spinal hydatid cysts are located most commonly at the thoracic spine (52\%), followed by the lumbar (37\%) and then the cervical and sacral levels. ${ }^{8}$ However, it remains controversial as to which region is more commonly involved. ${ }^{2}$ Braithwaite and Lees ${ }^{9}$ classified spinal hydatid cysts into five radiologic types: intramedullary, intradural-extramedullary, extradural, hydatid cyst of vertebrae, and paravertebral lesions extending to spinal structures.

Recurrence remains a major problem in spinal hydatidosis, with the literature citing rates of $30 \%$ to $100 \%{ }^{2,10}$ Given this high rate of recurrence, spinal hydatidosis has a poor prognosis and has been compared to spinal malignancy. ${ }^{8}$ A possible reason is the poor penetration of anthelminthic drugs to an intradural or extradural cyst. ${ }^{11}$

Early detection can offer timely treatment and prevent long-term sequelae. While radiographs can detect pedicle erosion and loss of vertebral body height, myelography can puncture the cyst and lead to intradural spread of the disease and anaphylaxis. Therefore, an MRI remains the investigation of choice and the appearance of a hydatid cyst on the scan is quite characteristic. It has two dome-shaped ends, with no debris in its lumen and looks like a sausage. Cyst walls are very thin and regular with no septation. ${ }^{12}$ Though serology may help in diagnosis, it is often not reliable.

The treatment of choice is surgical excision for neural decompression, and to establish the diagnosis. En masse excision of the spinal lesion largely depends on the location and the extent of the lesion. ${ }^{13}$ The cysts should ideally be removed into taking care not to rupture the cyst wall. Irrigating the wound with hypertonic saline or diluted povidone iodine solution after the cyst removal helps to destroy the parasites. ${ }^{8}$

The type of surgical procedure, extent of resection and decision whether to perform spinal stabilization or not depends on the site and extent of the disease, bone involvement and destruction 
causing spinal instability. ${ }^{13-15}$ In the early post-operative stage, adjuvant anthelminthic drug therapy must be given. The appropriate duration of anthelminthic therapy has not been established, and varies in literature from three months to two years. ${ }^{2,8,11,15}$

CONCLUSION: Although hydatid disease of the bone is extremely rare, the most common site is in the spine. There are no typical signs or symptoms of spinal hydatid disease other than those due to compression of the spinal cord. The best imaging modality is an MRI and the treatment of choice is surgery. A strict follow-up with regular MRI scans is mandatory during the post-operative period in order to ensure early detection of any recurrence. This is challenging to treat and can be prevented by removal of the cyst in toto and immediate postoperative anthelminthic drug therapy.

\section{REFERENCES:}

1. Charles RW, Govender S, Naidoo KS. Echinococcal infection of the spine with neural involvement. Spine (Phila Pa 1976). 1988 Jan; 13 (1):47-9.

2. Sharma NK, Chitkara N, Bakshi N, Gupta P. Primary spinal extradural hydatid cyst. Neurol India. 2003 Mar; 51 (1):89-90.

3. Rao S, Parikh S, Kerr R. Echinococcal infestation of the spine in North America. Clin Orthop Relat Res. 1991 Oct; (271):164-9.

4. Ferrandez HD, Gomez-Castresana F, Lopez-Duran L, Mata P, Brandau D, Sanchez-Barba A. Osseous hydatidosis. J Bone Joint Surg Am. 1978 Jul; 60(5):685-90.

5. Kahilogullari G, Tuna H, Aydin Z, Colpan E, Egemen N. Primary intradural extramedullary hydatid cyst. Am J Med Sci. 2005 Apr; 329(4):202-4.

6. Baysefer A, Gönül E, Canakçi Z, Erdoğan E, Aydoğan N, Kayali H. Hydatid disease of the spine. Spinal Cord. 1996 May; 34 (5):297-300.

7. Karadereler S, Orakdögen M, Kiliç K, Ozdogan C. Primary spinal extradural hydatid cyst in a child: case report and review of the literature. Eur Spine J. 2002 Oct; 11(5):500-3.

8. Schnepper GD, Johnson WD. Recurrent spinal hydatidosis in North America. Case report and review of the literature. Neurosurg Focus. 2004 Dec 15; 17(6):E8.

9. Braithwaite PA, Lees RF. Vertebral hydatid disease: radiological assessment. Radiology. 1981 Sep; 140 (3):763-6.

10. Bhake A, Agrawal A. Hydatid disease of the spine. J Neurosci Rural Pract. 2010 Jul; 1 (2):61-2.

11. Takayanagui OM, Bonato PS, Dreossi SA, Lanchote VL. Enantioselective distribution of albendazole metabolites in cerebrospinal fluid of patients with neurocysticercosis. $\mathrm{Br} \mathrm{J}$ Clin Pharmacol. 2002 Aug; 54 (2):125-30.

12. El Quessar A, Jroundi L, Tizniti S, Cissé M, Chakir N, El Hassani MR, Benchaâboun H, Jiddane M. CT and MRI features of spinal hydatidosis. A report of 8 cases. J Radiol. 2001 Aug; 82(8):91721.

13. Khazim RM. Spinal hydatid disease. South Med J. 2006 Feb; 99 (2):114.

14. Pamir MN, Ozduman K, Elmaci I. Spinal hydatid disease. Spinal Cord. 2002 Apr; 40 (4):153-60.

15. Fiennes AG, Thomas DG. Combined medical and surgical treatment of spinal hydatid disease: a case report. J Neurol Neurosurg Psychiatry. 1982 Oct; 45 (10):927-30. 


\section{CASE REPORT}

Figure 1A and 1B: MR imaging demonstrated extradural and extra spinal fluid filled mass with involvement of body, pedicle and posterior arch of D7 vertebra suggestive of neoplasm or infective process (TB)

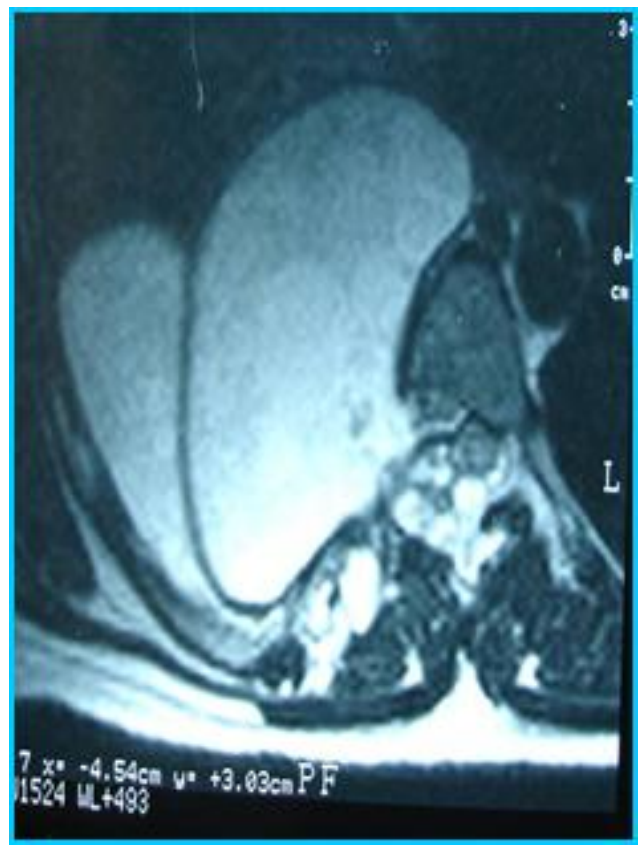

Figure 1A

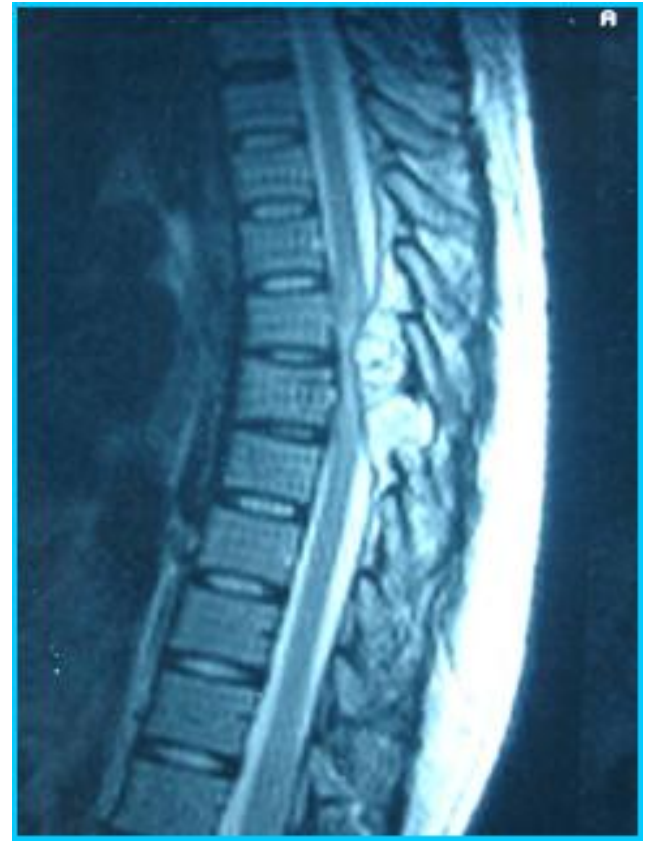

Figure 1B

Figure 2: Discharging sinus over the 9 th rib at one month post-operative follow-up

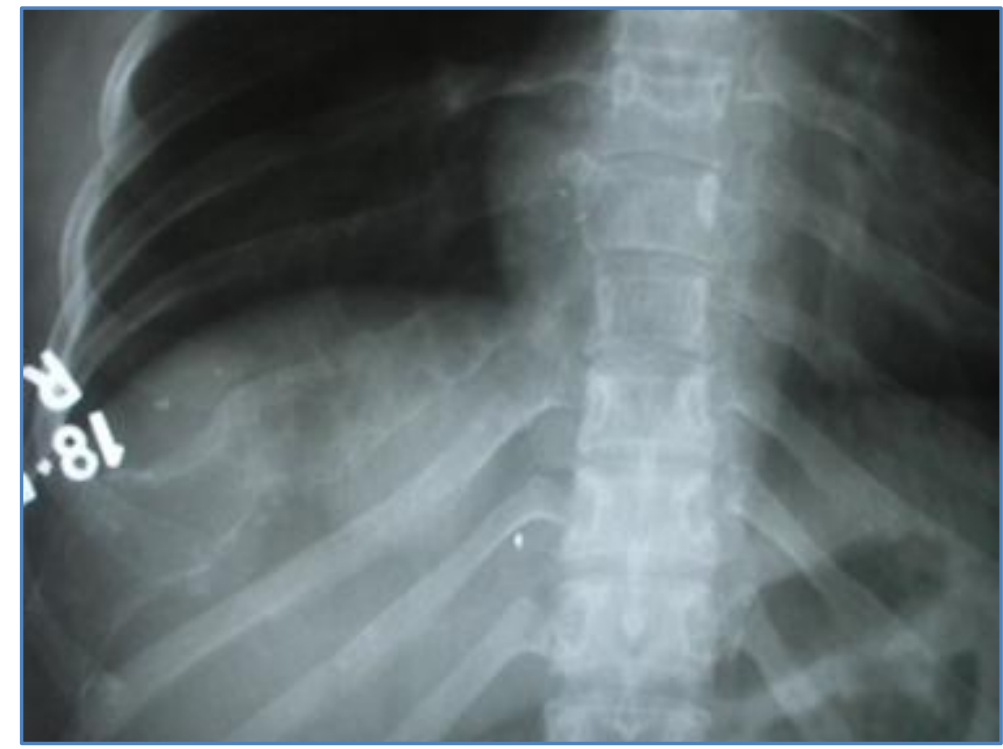

Figure 2 


\section{CASE REPORT}

Figure 3A and 3B: MRI at 3 months post-operative follow-up when she presented with mild degree fever. Para vertebral cystic cavities with no intra-spinal collection and involvement of three vertebrae.
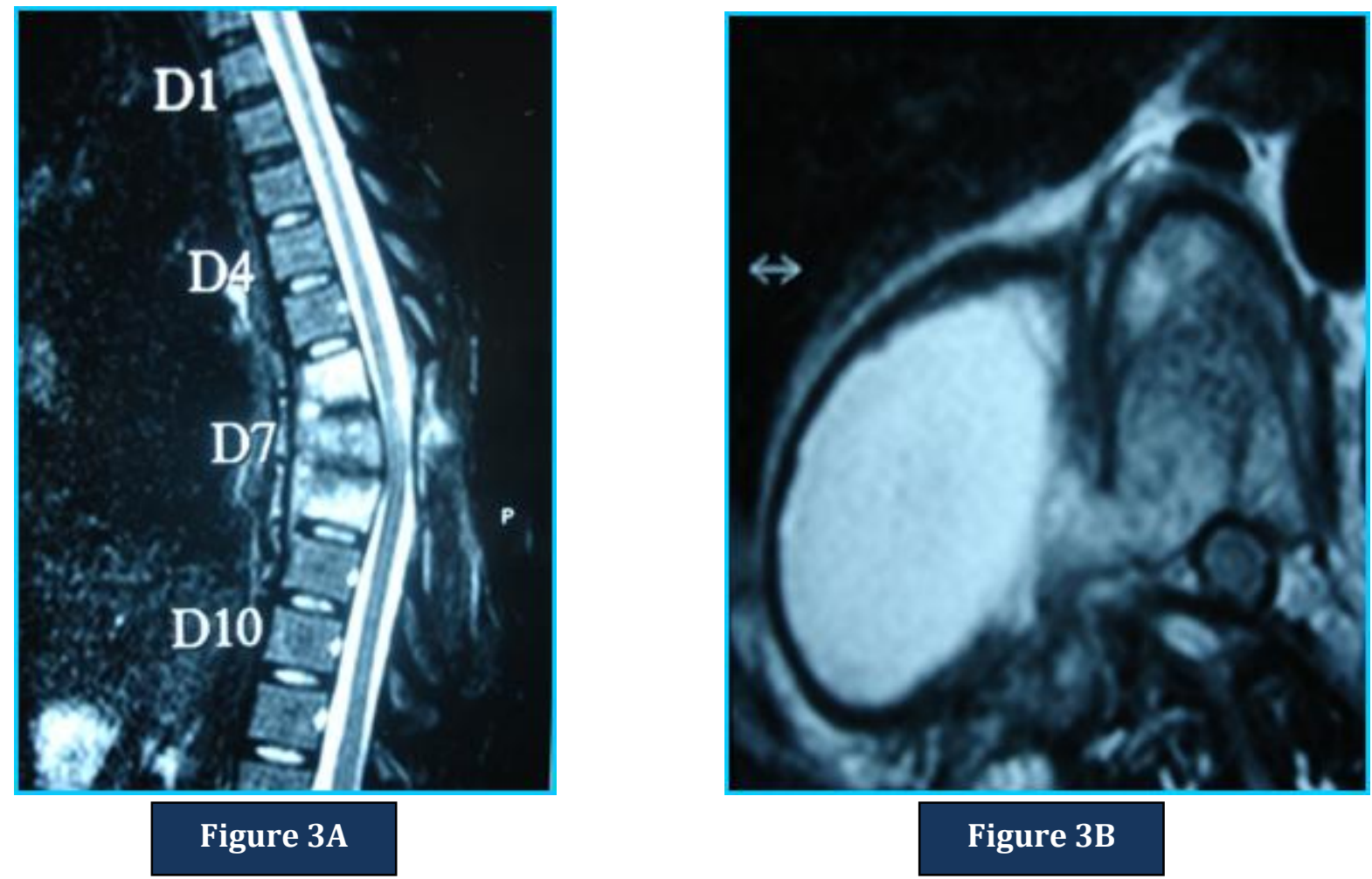

Figure 4A and 4B: MRI after 2 years showed cysts in the canal which was compressing the cord at D 5 \& D 6 level

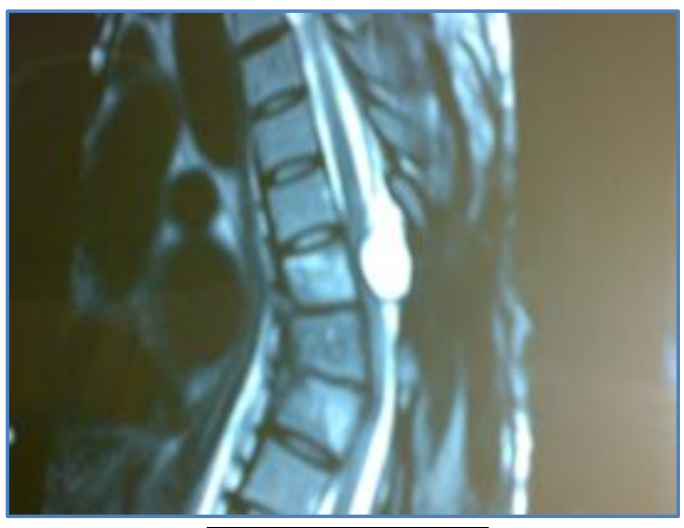

Figure 4A

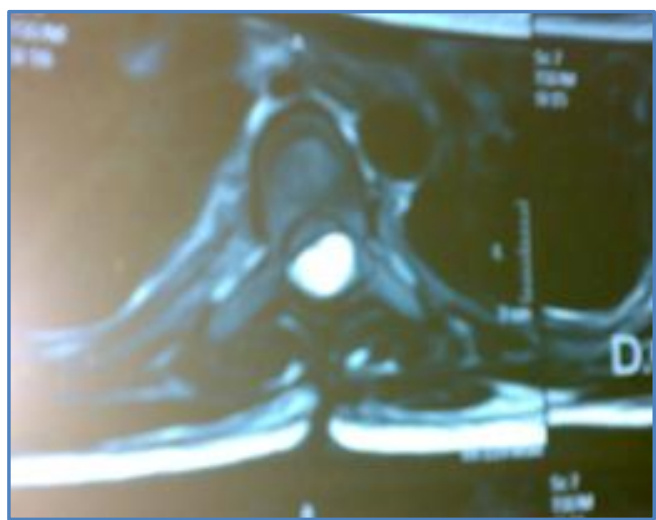

Figure 4B 


\section{CASE REPORT}

\section{AUTHORS:}

1. Avinash Parthasarathy

2. Chidanand K. J. C.

3. Prakashappa T. H.

\section{PARTICULARS OF CONTRIBUTORS:}

1. Assistant Professor, Department of Orthopaedics, Sanjay Gandhi Institute of Trauma and Orthopaedics.

2. Assistant Professor, Department of Orthopaedics, Sanjay Gandhi Institute of Trauma and Orthopaedics.

3. Professor, Department of Orthopaedics, Sanjay Gandhi Institute of Trauma and Orthopaedics.

\section{NAME ADDRESS EMAIL ID OF THE CORRESPONDING AUTHOR:}

Dr. Chidanand K. J. C,

Assistant Professor,

Department of Orthopaedics,

Sanjay Gandhi Institute of Trauma and

Orthopedics, Byrasandra,

Jayanagar IV 'T' Block, Bangalore - 560011.

Email: drchidanandkjc@gmail.com

Date of Submission: 29/04/2014. Date of Peer Review: 30/04/2014.

Date of Acceptance: 08/05/2014.

Date of Publishing: 19/05/2014. 